\title{
Caracterización del aceite de germen de trigo extraído con dióxido de carbono líquido y supercrítico
}

\author{
Por A. Molero Gómez*, M. D. Gordillo Romero y E. Martínez de la Ossa \\ Departamento de Ingeniería Química, Tecnología de Alimentos y Tecnologías del Medio Ambiente. \\ Universidad de Cádiz. Apdo. 40. E-11510 Puerto Real (Cádiz). Spain.
}

\section{RESUMEN}

\begin{abstract}
Caracterización del aceite de germen de trigo extraído con dióxido de carbono líquido y supercrítico.

En el presente trabajo se realiza un estudio de la calidad del aceite de germen de trigo obtenido mediante la extracción con dióxido de carbono a alta presión. En las condiciones operativas óptimas del proceso, el rendimiento de la extracción con dióxido de carbono líquido y supercrítico es similar al de la extracción con disolventes orgánicos, si bien la calidad del aceite obtenido es superior.

De ello se desprende el hecho de que el proceso de extracción utilizando dióxido de carbono, en condiciones sub- y supercríticas, puede resultar competitivo con el convencional al simplificar sustancialmente las etapas de refinado del aceite y eliminar la destilación del disolvente, las más costosas desde el punto de vista energético.
\end{abstract}

PALABRAS-CLAVE: Aceite de germen de trigo - Ácido graso insaturado - Calidad - Extracción supercrítica.

\section{SUMMARY}

Characterization of wheat germ oil extracted by liquid and supercritical carbon dioxide.

In this work the quality of wheat germ oil obtained by extraction with liquid and supercritical carbon dioxide was studied. At the optimum operating conditions, the yield of supercritical extraction is similar to the conventional extraction by organic solvent, but the quality of the wheat germ oil extracted by supercritical carbon dioxide is higher.

Furthermore, supercritical extraction could be more economical than organic extraction because the solvent removal by distillation is not necessary and several steps of the following oil refining process can be simplified.

KEY-WORDS: Quality - Supercritical extraction Unsaturated fatty acid - Wheat germ oil.

\section{INTRODUCCIÓN}

Desde hace algunos años son bien conocidos los efectos beneficiosos que los aceites de origen vegetal ejercen sobre el ser humano. Este hecho se debe, fundamentalmente, al elevado contenido en ácidos grasos insaturados y a su alto valor energético (Morand y Silvestre, 1960; Elder y Rathman, 1962; Bertoni y col., 1966). Así, determinados ácidos grasos y sus glicéridos juegan un papel muy importante como constituyentes de estructuras y membranas celulares, como precursores de diversas biomoléculas y como controladores del contenido lipídico en sangre y del nivel de colesterol en la misma (Cantafora, 1982).

La dieta mediterránea ha hecho de España uno de los principales países productores y consumidores de aceites de origen vegetal, especialmente de aceite de oliva y girasol. Sin embargo, las cada vez mayores restricciones que la actual Legislación establece acerca de la completa eliminación de los disolventes orgánicos empleados en la extracción de productos destinados a consumo humano (entre los que se incluyen los aceites de semilla), hace necesaria la búsqueda de técnicas de extracción alternativas a los métodos convencionales ya existentes. Por otro lado, y dado los recientes reveses sufridos en relación a la producción de aceite de oliva y a la actual situación socioeconómica de España, parece necesaria también la búsqueda de otras materias primas vegetales que permitan obtener aceites de gran calidad que sean competitivos con los ya existentes en el mercado.

En lo que se refiere a la búsqueda de técnicas alternativas de extracción, la Extracción Supercrítica (ESC) utilizando dióxido de carbono como disolvente, se ha revelado como una de las técnicas alternativas capaces de sustituir a la extracción convencional con disolventes orgánicos (Martínez de la Ossa y Galán, 1991). Entre sus muchas ventajas, la ESC ofrece rendimientos de extracción próximos a los obtenidos en la extracción convencional; además, y en contraste con los disolventes orgánicos, el dióxido de carbono no deja rastros en el producto extraído, no es tóxico, ni inflamable, ni corrosivo, es barato y fácilmente disponible en grandes cantidades y con un alto grado de pureza. Finalmente, el dióxido de carbono presenta una baja presión y temperatura críticas $\left(73,8 \mathrm{~atm}\right.$. y $31,1^{\circ} \mathrm{C}$, respectivamente), por lo que es idóneo para el tratamiento de productos termolábiles, muy comunes en la industria alimentaria. 
En lo relativo a la búsqueda de nuevas materias primas, el germen de trigo presenta una alta relación de ácidos grasos insaturados, siendo el linoleico mayoritario. Además, el germen de trigo es una de las materias primas vegetales con mayor contenido en vitamina $E$ (Saito y Yamauchi, 1990) cuya positiva acción en el ser humano está ampliamente descrita en la bibliografía (Rustan y col., 1993; Acilu y Font, 1994). Actualmente, gran parte de la producción de vitamina $E$ se realiza a partir de desodorizados de aceite provenientes del proceso de refinado (Saito y col., 1989; Lee y col., 1991).

En el presente trabajo se ha llevado a cabo el estudio de la calidad del aceite de germen de trigo obtenido por extracción con dióxido de carbono líquido y supercrítico, en las condiciones operativas óptimas del proceso desde el punto de vista del rendimiento de la ESC. Además, el trabajo se ha planteado en base a la comparación de las calidades de ambos aceites, con la idea de comprobar si el proceso de ESC puede ser económicamente competitivo cuando se compara con la extracción con disolventes orgánicos.

\section{MATERIAL Y MÉTODOS}

Como materia prima para los procesos de extracción se ha utilizado germen de trigo $(0,75 \mathrm{~mm}$ de diámetro medio) suministrado por las instalaciones que la empresa CODEC, S.A. tiene en Valladolid.

El equipo utilizado para realizar los procesos de ESC con dióxido de carbono, así como el protocolo de operación se ha descrito detalladamente en un trabajo previo (Molero y col., 1995).

La extracción convencional con disolventes líquidos se llevó a cabo utilizando hexano en un aparato tipo "SOXHLET», durante 16 horas, con el fin de garantizar el máximo rendimiento extractivo y poder establecer la base comparativa para el proceso de ESC.

La caracterización físico-química del aceite extraído se ha llevado a cabo siguiendo las normas que la A.O.A.C. (1980) establece y que han de analizarse para todos aquellos aceites destinados a consumo humano (UNE-55-022-78). Los parámetros analizados fueron: índice de refracción, densidad, viscosidad y absorbancia a $290 \mathrm{~nm}$. Los distintos índices que se determinaron fueron: acidez (expresado en tanto por ciento de ácido oleico), yodo (método de Hanus), saponificación y peróxidos. También se determinó el porcentaje de insaponificables.

La composición del aceite en ácidos grasos se ha realizado, previa metilación de los mismos (Molero, 1994), por cromatografía gaseosa usando un cromatógrafo «HEWLETT-PACKARD» (Pittsburgh, PA, USA) modelo «5890», con un software de integración «Hewlett-Packard ChemStation» versión «A.02.01», provisto de una columna capilar "Carbowax $20 \mathrm{M}$ » (Supelco, Bellefonte, PA, USA) y un detector FID.

Todos los productos y reactivos químicos empleados son de pureza «para análisis». La pureza del dióxido de carbono utilizado fue del 99,95\% («Carburos Metálicos»).

\section{DISCUSION DE RESULTADOS}

\subsection{Rendimiento de extracción}

En la Tabla I se presentan los valores experimentales de los rendimientos de los procesos extractivos realizados sobre el germen de trigo, tanto con dióxido de carbono líquido y supercrítico como con hexano, en las condiciones óptimas de acondicionamiento de la materia prima, así como operativas de ambos procesos.

Tabla I

Rendimientos de extracción utilizando $\mathrm{CO}_{2}$ a altas presiones y hexano como disolventes

\begin{tabular}{cc}
\hline Condiciones de operación & Rendimiento (\% en peso) \\
\hline $100 \mathrm{bar}, 10^{\circ} \mathrm{C}, 1,5 \mathrm{~L} / \mathrm{min}, 3 \mathrm{~h}$. & 7,3 \\
$100 \mathrm{bar}, 20^{\circ} \mathrm{C}, 1,5 \mathrm{~L} / \mathrm{min}, 3 \mathrm{~h}$. & 7,6 \\
$150 \mathrm{bar}, 30^{\circ} \mathrm{C}, 1,5 \mathrm{~L} / \mathrm{min}, 3 \mathrm{~h}$. & 7,5 \\
$150 \mathrm{bar}, 40^{\circ} \mathrm{C}, 1,5 \mathrm{~L} / \mathrm{min}, 3 \mathrm{~h}$. & 8,0 \\
$200 \mathrm{bar}, 50^{\circ} \mathrm{C}, 1,5 \mathrm{~L} / \mathrm{min}, 3 \mathrm{~h}$. & 8,0 \\
$200 \mathrm{bar}, 60^{\circ} \mathrm{C}, 1,5 \mathrm{~L} / \mathrm{min}, 3 \mathrm{~h}$. & 7,8 \\
Soxhlet, $16 \mathrm{~h} ., 65^{\circ} \mathrm{C}$ & 8,6
\end{tabular}

Como puede comprobarse, el rendimiento de la ESC del aceite de germen de trigo es ligeramente inferior al de la extracción con hexano. No obstante, este hecho ha sido recogido por otros autores cuando han comparado los rendimientos obtenidos por la ESC frente a los obtenidos en dispositivos tipo Soxhlet para distintas materias primas vegetales (Stahl y col., 1980; Friedrich y List, 1982; Taniguchi y col., 1985; Zhao y col., 1987, Molero y col., 1995). La explicación de este fenómeno es la mayor selectividad que presenta el $\mathrm{CO}_{2}$ a altas presiones. Así, los disolventes orgánicos empleados en la extracción convencional no son selectivos de triglicéridos, en tanto que si lo son de ácidos grasos libres, fosfolípidos, pigmentos, y diversos complejos lipídicos además del aceite (glicolípidos, en el caso del hexano); en cambio, el $\mathrm{CO}_{2}$ supercrítico sí es un disolvente selectivo en relación a los triglicéridos, que, además, no extrae estos complejos lipídicos.

De los resultados mostrados en la Tabla I, no existen diferencias apreciables, en lo que a rendimiento se refiere, cuando se compara la ESC utilizando dióxido de carbono líquido y supercrítico frente a la extracción en dispositivos Soxhlet utilizando hexano como disolvente. Llegados a este punto, y admitiendo de antemano que la ESC pudiera desplazar a las técnicas de extracción convencionales, la elección de las condiciones óptimas de operación utilizando dióxido de carbono líquido y supercrítico habría que buscarlas atendiendo a consideraciones económicas y, como se expondrá a continuación, desde el punto de vista de la calidad de los aceites obtenidos. 


\section{2. Índices físico-químicos}

En la Tabla II se recoge la caracterización físicoquímica de los aceites de germen de trigo obtenidos mediante ESC utilizando dióxido de carbono líquido y supercrítico, así como el obtenido mediante dispositivos Soxhlet utilizando hexano como disolvente. El análisis de estos parámetros permitirá analizar la calidad de los distintos aceites y seleccionar las condiciones óptimas del proceso de ESC.

A tenor de los resultados obtenidos, puede deducirse que la combinación de la presión y temperatura de operación utilizadas durante la extracción con dióxido de carbono a alta presión juegan un papel determinante en la calidad de los aceites obtenidos, ya que existen diferencias apreciables entre los distintos índices (salvo en los de refracción, densidad y viscosidad) para los distintos tipos de aceites.

La acidez presenta una tendencia parabólica alcanzando valores mínimos cuando la ESC se llevó a cabo a temperaturas de 30 y $40^{\circ} \mathrm{C}$. Sin embargo, para el resto de las condiciones de operación de la ESC y para la extracción con hexano, los valores alcanzados para el índice de acidez son muy elevados. La diferencia de valores encontrada puede deberse a diversos factores:

- En primer lugar, las ESC realizadas a temperaturas de 30 y $40^{\circ} \mathrm{C}$, le confieren al $\mathrm{CO}_{2}$ una alta especificidad por los triglicéridos contenidos en el aceite de germen de trigo, siendo mínima la cantidad de ácidos grasos libres extraídos.

- Por el contrario, cuando la extracción a alta presión se realizó a bajas $\left(10\right.$ y $\left.20^{\circ} \mathrm{C}\right)$ y altas temperaturas $(50$ y $60^{\circ} \mathrm{C}$ ), el dióxido de carbono pierde gran parte de su selectividad, comportándose de forma análoga a cualquier otro disolvente líquido, incrementándose como consecuencia el valor del citado índice.

- Finalmente, el empleo de temperaturas moderadas (de hasta $40^{\circ} \mathrm{C}$ ) no debe provocar la ruptura de los triglicéridos durante el proceso de extracción, a diferencia de la extracción llevada a cabo en dispositivos Soxhlet utilizando hexano como disolvente. Por ello, cabe pensar que el germen de trigo es de por sí una materia vegetal con un elevado contenido en ácidos grasos libres, a diferencia de la semilla de uva (Molero y col., 1995). Sin embargo, no existen datos en la bibliografía referidos al germen de trigo y a la ESC que respalden los datos de acidez obtenidos.

Independientemente del dato de acidez obtenido para cada una de las condiciones de extracción, si se descuenta el contenido de ácidos grasos libres en los aceites procedentes de los dos procesos, los valores de los índices de yodo y saponificación indican que la cantidad de triglicéridos presentes en los aceites extraídos en ambos procesos es similar, revelando que en ambas técnicas extractivas el germen de trigo queda totalmente agotado y que, en este sentido, no hay diferencia entre el proceso supercrítico y el convencional.

Los valores relativos de la fracción insaponificable sí muestran una clara diferencia entre los aceites extraídos con los dos disolventes. En este sentido, conforme aumenta la temperatura en el proceso de extracción a altas presiones, se favorece la extracción de los compuestos que constituyen la fracción insaponificable (Ohgaki y col., 1989). En lo que respecta a la extracción con hexano, el valor de insaponificables que se obtiene es muy bajo (similar al de las ESC conducidas a 10 y $20^{\circ} \mathrm{C}$ ) y en línea con el determinado para el aceite de semilla de uva (Molero y col., 1995). No hay que olvidar que estos productos suelen ser

Tabla II

Comparación de los aceites extraídos con $\mathrm{CO}_{2}$ a altas presiones y con hexano

\begin{tabular}{|c|c|c|c|c|c|c|c|}
\hline \multirow{3}{*}{$\begin{array}{l}\text { Parámetros } \\
\text { físico-químicos }\end{array}$} & \multicolumn{7}{|c|}{ Disolvente } \\
\hline & \multirow[b]{2}{*}{$10^{\circ} \mathrm{C}$} & \multicolumn{3}{|c|}{ dioxido de carbono } & \multirow[b]{2}{*}{$50^{\circ} \mathrm{C}$} & \multirow[b]{2}{*}{$60^{\circ} \mathrm{C}$} & \multirow{2}{*}{$\begin{array}{c}\text { hexano } \\
16 \mathrm{~h}, 65^{\circ} \mathrm{C}\end{array}$} \\
\hline & & $20^{\circ} \mathrm{C}$ & $30^{\circ} \mathrm{C}$ & $40^{\circ} \mathrm{C}$ & & & \\
\hline Índice de refracción & 1,475 & 1,474 & 1,476 & 1,477 & 1,476 & 1,475 & 1,472 \\
\hline Densidad & 0,919 & 0,918 & 0,920 & 0,918 & 0,916 & 0,918 & 0,905 \\
\hline Viscosidad $\left(c p, 30^{\circ} \mathrm{C}\right)$ & 46 & 45 & 40 & 38 & 46 & 35 & 30 \\
\hline Absorb. 290 nm (IgE) & 0,676 & 0,665 & 0,757 & 1,157 & 0,841 & 0,878 & 0,969 \\
\hline Índice de acidez & 31,6 & 34,9 & 14,0 & 12,4 & 25,3 & 34,4 & 27,3 \\
\hline Índice de yodo & 111 & 112 & 102 & 100 & 107 & 115 & 107 \\
\hline Índice de saponif. & 217 & 181 & 185 & 185 & 197 & 204 & 207 \\
\hline Insaponificables (\%) & 1,59 & 2,20 & 5,88 & 7,28 & 6,66 & 7,05 & 2,76 \\
\hline Índice de peróxidos & 73 & 69 & 75 & 95 & 98 & 93 & 147 \\
\hline
\end{tabular}


fácilmente degradables, y que la temperatura de operación (especialmente alta en el caso del hexano) puede afectarles sensiblemente.

Con respecto a los parámetros de absorbancia en la región del UV y al índice de peróxidos, ambos son indicativos de la degradación del aceite, y, especialmente, de la oxidación de determinados compuestos (un ejemplo claro son los tocoferoles). A tenor de los resultados se pueden establecer las siguientes conclusiones:

- Con respecto a la absorbancia en la región UV, la norma U.N.E. correspondiente establece que se ha de medir a dos longitudes de onda características: 232 y $270 \mathrm{~nm}$. La primera de ellas es indicativa de la oxidación del ácido linoleico (el cual contiene un sistema diénico cuya oxidación origina un hidro-peróxido que absorbe a la citada longitud de onda), en tanto que la segunda es indicativa del alcance del grado de oxidación del aceite y es más fiable que la primera. Experimentalmente se ha encontrado un único máximo de absorción en torno a los $290 \mathrm{~nm}$ para todos los aceites, si bien la propia norma U.N.E. advierte que el máximo de absorción a $270 \mathrm{~nm}$ puede encontrarse desplazado a longitudes de onda mayores en algunos casos.

- En lo que se refiere a los valores encontrados para el índice de peróxidos, los datos reflejan que el grado de degradación encontrado es muy similar salvo para el aceite extraído utilizando hexano como disolvente. En lo que se refiere a los aceites extraídos a altas presiones, los valores obtenidos son muy inferiores a los encontrados para el aceite de semilla de uva extraídos con dióxido de carbono supercrítico en un trabajo previo (Molero y col., 1995).
- En función de los resultados de ambos parámetros puede concluirse que el grado de oxidación de los lípidos presentes en el aceite de germen de trigo es mínimo (no existe absorbancia a $232 \mathrm{~nm}$ ) y que la única degradación existente es posiblemente debida a la oxidación de determinados compuestos presentes en la fracción insaponificable registrada en torno a los $290 \mathrm{~nm}$.

A tenor del análisis de los resultados de los parámetros físico-químicos del aceite de germen de trigo, y si se tiene presente que entre los objetivos del posterior proceso de refinado del aceite, en el caso de la extracción líquida, se contempla la reducción a valores aceptables de los contenidos de insaponificables y ácidos grasos libres, se puede deducir que, en el caso de la extracción supercrítica realizada a 150 bar y $40^{\circ}$ C, esta etapa podría simplificarse considerablemente dado el bajo contenido del aceite en estos componentes indeseables. Además, el proceso de desodorización proporcionaría una fracción concentrada de tocoferoles, de gran aplicación en la industria alimentaria y farmacéutica.

\subsection{Composición en ácidos grasos}

En la Tabla III se recogen las composiciones en ácidos grasos de los aceites extraídos mediante los dos procesos estudiados. Como puede observarse, el contenido en ácidos grasos del aceite de germen de trigo extraído con $\mathrm{CO}_{2}$ supercrítico es muy similar al extraído con hexano. Hay que destacar su elevado contenido en ácidos grasos inaturados frente a la baja proporción de ácidos grasos saturados, lo cual lo hace muy adecuado para su uso en alimentación.

Tabla III

Composición en ácidos grasos del aceite de germen de trigo extraído con dióxido de carbono líquido y supercrítico así como con hexano en dispositivos Soxhlet

\begin{tabular}{lcccccc}
\hline & \multicolumn{7}{c}{ Composición en ácidos grasos (\% en peso) } \\
\cline { 2 - 7 } Condiciones de operación & $\mathbf{C 1 6 : 0}$ & $\mathbf{C 1 6 : 1}$ & $\mathbf{C 1 8 : 0}$ & $\mathbf{C 1 8 : 1}$ & $\mathbf{C 1 8 : 2}$ & $\mathbf{C 1 8 : 3}$ \\
\hline $100 \mathrm{bar}, 10^{\circ} \mathrm{C}$ & 18,91 & 0,24 & 0,69 & 16,41 & 57,26 & 6,48 \\
$100 \mathrm{bar}, 20^{\circ} \mathrm{C}$ & 18,95 & 0,23 & 0,73 & 16,52 & 57,10 & 6,46 \\
$150 \mathrm{bar}, 30^{\circ} \mathrm{C}$ & 18,21 & 0,22 & 0,52 & 14,17 & 58,40 & 8,49 \\
$150 \mathrm{bar}, 40^{\circ} \mathrm{C}$ & 18,15 & 0,20 & 0,52 & 13,94 & 58,56 & 8,25 \\
$200 \mathrm{bar}, 50^{\circ} \mathrm{C}$ & 18,25 & 0,24 & 0,59 & 14,82 & 58,64 & 7,47 \\
$200 \mathrm{bar}, 60^{\circ} \mathrm{C}$ & 18,48 & 0,22 & 0,49 & 13,81 & 58,74 & 8,25 \\
Soxhlet, $16 \mathrm{~h} ., 65^{\circ} \mathrm{C}$ & 18,09 & 0,22 & 0,50 & 13,69 & 58,99 & 8,51 \\
\hline
\end{tabular}


Por otra parte, y como ocurre en el caso del aceite de semilla de uva, el ácido graso mayoritario es el linoleico (C18:2), con un 55\% del total. Este ácido graso insaturado, al no poder ser sintetizado a partir de ningún otro ácido graso ni de ninguna otra fuente por la célula y tejidos celulares, debe ser ingerido en la dieta (Carola, 1974; Cantafora, 1982). Entre todas las acciones en las que interviene dicho ácido graso, cabe destacar: su efecto protector frente a la arteriosclerosis y a la trombosis (Williams, 1980; Connor y Connor, 1990); su acción protectora frente a las afecciones cardiovasculares (Kummerow, 1975; Williams, 1980; Hirai y col., 1987); como agente hipocolesterémico (Horrobin y Manku, 1983); como precursor del ácido araquidónico; el cual origina a su vez las prostaglandinas (Mancha, 1976; Williams, 1980); y como principal agente en la regeneración del hígado (Ciangherotti y col., 1984). Si atendemos a todas estas razones, el aceite de germen de trigo puede constituir una fuente de primer orden en lo que a producción de ácido linoleico se refiere.

\section{CONCLUSIONES}

La calidad del aceite de germen de trigo obtenido por extracción supercrítica con dióxido de carbono a 150 bar y $40^{\circ} \mathrm{C}$ es superior a la encontrada cuando se emplearon otras condiciones de operación y a la del que se obtiene por extracción con disolventes líquidos, sin refinar. De hecho, su calidad se aproxima a la del aceite procedente del proceso convencional, una vez refinado, y podría ser idéntica si la despresurización para eliminar el disolvente se realizase progresivamente.

Es más, dado que el proceso con dióxido de carbono tiene lugar a temperatura ambiente, otros componentes no sufren reacciones secundarias de degradación (como es el caso de los tocoferoles), lo cual permitiría la obtención de concentrados de tocoferol provenientes de la etapa de desodorización durante el transcurso de proceso de refinado.

La completa separación del disolvente supercrítico y el aceite durante la despresurización hace innecesaria cualquier otra operación posterior de eliminación del disolvente, no quedando rastros de disolvente en el aceite extraído (garantizándose así la calidad del producto extraído). De esta forma, en relación al proceso convencional con disolventes líquidos, el proceso supercrítico elimina una etapa muy costosa energéticamente, la destilación el disolvente, y simplifica el posterior refinado del aceite.

Por todo lo anterior, y a pesar de la necesidad de trabajar a altas presiones, la extracción supercrítica aplicada a la obtención de aceite de germen de trigo ( $y$, en general, a otras materias vegetales), puede resultar económicamente competitiva con la extracción líquida, máxime si se tiene en cuenta la obtención de otros productos de alto valor añadido contenidos en el aceite, como es el caso de los tocoferoles.

\section{REFERENCIAS}

A.O.A.C. (ASSOCIATION OF OFFICIAL AND ANALYTICAL CHEMISTS'SOCIETY) (1980). - «Standar Methods of Analysis".- 13 th Edition. Washington D. C.

Acilu, M.; Font, J. (1994). - «Identificación de isómeros de tocoferol o vitamina $E$ en muestras de aceites vegetales por HPLC con detector de fotodiodos"Cromatografía,196, 866-869.

Bertoni, M. H.; Karman de Sutton, G.; Cattaneo, P. (1966). - Aceites de Semilla de Uva Argentina. Composición Química".- Anales Asoc. Quím. Argentina, 54, 89-99.

Elder, A. L., Rathman, D. M. (1962). -Econ. Botany, 16, 196

Cantafora, A. (1982). - «Ruollo dell'Acido Linoleico nell'Alimentazione Umana: Aspetti Teorici e Pratici».Industrie Alimentari, 21, 454-458.

Carola, C. (1974). - «ll Fabbisogno Lipidico dell'Uomo: Proposte per un Oilo Equilibrato".- Riv. Ital. Sostanze Grasse 51, 353-361.

Ciangherotti, S.; Caponneto, A.; Lantieri, P. B., Rondinone, R. (1984). - “L'Acido Linoleico nella Rigenerazione del Fegato»-. Riv. Ital. Sostanze Grasse, 51, 345-347.

Connor, W. E.; Connor, S. L. (1990). - «Diet, Atherosclerosis, and Fish Oil»-. Adv. Intern. Med., 35, 139-172.

Friedrich, J. P.; List, G. R. (1982). - "Characterization of Soybean Oil Extracted by Supercritical Carbon Dioxide and Hexane»-. J. Agric. Food Chem., 30, 192-193.

Hirai, A.; Terano, T.; Saito, H.; Tamura, Y.; Yoshida, S. (1987). - «Clinical and Epidemiological Studies of Eicosapentaenoic acid in Japan»-. In Proceedings of the AOCS Short Course on Polyunsaturated Fatty Acids and Eicosanoids, ed. W. E. M. Lands, Champaign, Illinois, USA. 9-24.

Horrobin, D.; Manku, M. (1983). -«How do Polyunsaturated Fatty Acids Lower Plasma Cholesterol Levels?»Lipids, 18 (8), 558-562.

Kummerow, F. A. (1975). - «Lipids in Atherosclerosis»-. J. Food Science, 40, 12-17.

Lee, H.: Chung, B. H.; Park, Y. H. (1991). - «Concentration of Tocopherols from Soybean Sludge by Supercritical Carbon Dioxide»- J.A.O.C.S., 68 (8), 571-573.

Mancha, M. (1976). - «Biosíntesis de los Ácidos Grasos en las Plantas"-, Grasas y Aceites, 27 (1), 33-39.

Martínez de la Ossa, E.; Galán, M. A. (1991). - «Extracción con Fluidos Supercríticos. (III). Aplicaciones".Ingeniería Química, 262, 251-260.

Molero, A. (1994). -Tesis de Licenciatura-. Universidad de Cádiz.

Molero, A.; Pereyra, C.; Martínez de la Ossa, E. (1995). - «Caracterización del aceite de semilla de uva extraído con dióxido de carbono supercrítico"-Grasas y Aceites, 46, 29-34.

Morand, P.; Silvestre, J. (1960). - «Contribution a l'Etude d'une Huile Végétale a base d'Acides Gras Polyinsatures: l'Huile de Pépin de Raisin. Caracteres Analytiques, Qualités Alimentaires et Dietetiques»-Ann. Fals. Exp. Chim. 53, 193-203.

Ohgaki, K.; Tsukahara, I.; Semba, K.; Katayama, T. (1989). «A fundamental study of extraction with a supercritical fluid. Solubilities of $a$-tocopherol, palmitic acid, and tripalmitin in compressed carbon dioxide at $25^{\circ} \mathrm{C}$ and $40^{\circ}$ C»-. International Chemical Engineering, 2 (2), 302-308.

Rustan, I.; Damiano, M. A.; Lergards, G. (1993). - «Mise au point de la recherche d'antioxydants a usage alimentaire et applications".- Ann. Fals. Exp. Chim., 86 (919), 201. 214.

Saito, M.; Yamauchi, Y.; Inomata, K.; Kottkamp, W. (1989). - «Enrichment of Tocopherols in Wheat Germ by Directly 
Coupled Supercritical Fluid Extraction with Semipreparative Supercritical Fluid Chromatography".Journal of Chromatographic Science, 27, 79-85.

Saito, M.; Yamauchi, Y. (1990). - «Isolation of tocopherols from wheat germ oil by recicle semi-preparative supercritical fluid chromatography"-. Journal of Chromatography, 505, 257-271.

Stahl, E.; Schütz, E.; Mangold, K. (1980). -«Extraction of Seed Oils with Liquid and Supercritical Carbon Dioxide»- J. Agric. Food Chem., 28, 1153-1157.

Taniguchi, M.; Tsuji, T.; Shibata, M.; Kobayashi, T. (1985). - «Extraction of Oils from Wheat Germ with Supercritical Carbon Dioxide»- Agric. Biol. Chem., 49, (8), 23672372.

Williams, J. (1980). - «Progrès Récents de la Recherche sur les Acides Gras Essentiels».- Oléagineux, 35 (10), 457459.

Zhao, W.; Shisshikura, A.; Fujimoto, K.; Arai, K.; Saito, S. (1987). - «Fractional Extraction of Rice Bran Oil with Supercritical Carbon Dioxide"- Agric. Biol. Chem., 51 (7), 1773-1777.

Recibido: Noviembre 1996 Aceptado: Marzo 1997 